Methods In 2013 we reviewed clinical notes coded for herpes suppression to establish whether BASHH and local standards were met for management of herpes suppression and routine blood monitoring.

Results 41 cases were reviewed. 32 (73\%) had baseline blood tests. Of these 6/32 (19\%) had abnormal results: 2 raised LFTS, 2 low estimated Glomerular Filtration Rate (eGFR), 2 low neutrophils - all resolved on repeating except one with fluctuating neutropenia. 19/32 (47\%) had bloods repeated at our service and additional 16\% advised to attend GP. Only 1/19 (5\%) had normal baseline bloods, low eGFR at one month, but normal at 2 months.

\begin{tabular}{ll} 
Abstract P233 Table 1 & Auditable standard results \\
\hline Standards (Target: BASHH or *local) & Achieved \\
\hline Virological confirmation (100\%) & $98 \%$ \\
Viral typing (100\%) & $90 \%$ \\
Baseline FBC, U\&E, LFT ( $\left.{ }^{*} 100 \%\right)$ & $73 \%$ \\
Offer letter to GP (*100\%) & $78 \%$ \\
Letter to obstetrics if pregnant (*100\%) & $100 \%$ \\
\hline
\end{tabular}

Discussion 19\% of those tested had blood abnormalities at baseline, but only $3 \%$ had on-going abnormalities likely affected by acyclovir. We recommend checking U\&E, LFT and FBC at baseline. If normal no further monitoring is needed. If mildy abnormal repeat but continue aciclovir. If significantly low eGFR, leucopenia or elevated LFTs either dose reduce or stop acyclovir and investigate.

\section{P234 GLOBAL ESTIMATES OF PREVALENT AND INCIDENT HERPES SIMPLEX VIRUS TYPE 2 INFECTIONS IN 2012}

${ }^{1}$ Katharine Looker*, ${ }^{2}$ Amalia Magaret, ${ }^{1}$ Katherine Turner, ${ }^{1}$ Peter Vickerman, ${ }^{3}$ Sami Gottlieb, ${ }^{3}$ Lori Newman. 'University of Bristol, Bristol, UK; ${ }^{2}$ University of Washington, Seattle, USA; ${ }^{3}$ World Health Organization, Geneva, Switzerland

\subsection{6/sextrans-2015-052126.276}

Background/introduction Genital herpes, usually caused by infection with herpes simplex virus type 2 (HSV-2), can cause substantial morbidity in the form of painful genital ulcers in infected adults and adolescents, as well as significant psychosocial morbidity. Neonatal herpes, acquired during delivery from mothers with genital herpes, is rare but often fatal. Additionally, HSV-2 increases susceptibility to, and transmissibility of, HIV. The global burden of HSV-2 was last estimated for 2003.

Aim(s)/objectives To present new global HSV-2 estimates for 2012 for females and males aged 15-49 years.

Methods Literature review of HSV-2 prevalence studies worldwide since 2000 , followed by fitting of a model with constant HSV-2 incidence by age to pooled HSV-2 prevalence values by WHO region, age and sex. Prevalence values were adjusted for test sensitivity and specificity.

Results In 2012, we estimate that 417 million people aged 15-49 years (range: 274-678 million) had existing HSV-2 infection world-wide: a global prevalence of $11.3 \%$. Of those infected, 267 million were women. Also in 2012, we estimate that 19.2 million (range: 13.0-28.6 million) individuals aged 15-49 years were newly-infected with HSV-2: $0.5 \%$ of all individuals globally. Prevalence was highest in Africa (31.5\%), followed by the Americas (14.4\%). Burden of numbers infected was highest in Africa. However, despite lower prevalence, SouthEast Asia and Western Pacific regions also contributed large numbers to the global totals because of large population sizes.
Discussion/conclusion The global burden of HSV-2 infection is large, highlighting the critical need for development of vaccines, microbicides and other prevention strategies against HSV-2.

\section{P235 PREVALENCE AND RISK FACTORS ASSOCIATED WITH ORAL HPV AMONG STI CLINIC ATTENDEES}

${ }^{1}$ Karly Louie, ${ }^{1}$ Lesley Ashdown-Barr, ${ }^{1}$ Caroline Reuter, ${ }^{1}$ Attila Lorincz, ${ }^{1}$ Peter Sasieni, ${ }^{2}$ Jill Zelin*. 'Centre for Cancer Prevention, Wolfson Institute of Preventive Medicine, Queen Mary University of London, London, UK; ${ }^{2}$ St Bartholomew's Hospital, London, UK

\subsection{6/sextrans-2015-052126.277}

Background Oral human papillomavirus (HPV) infection increases the risk of a sub-set of head and neck cancers. The epidemiology of oral HPV infection is not well understood.

Aim To describe the prevalence and risk factors for oral HPV infection amongst STI clinic attendees.

Methods Participants were recruited from a STI clinic, completed a risk factor questionnaire and provided oral samples for HPV DNA testing by a highly sensitive PCR using the SPF-10 broad spectrum primers. Overall positivity (prevalence) for any HPV was calculated. Chi-square test was used to determine the association between risk factors and oral HPV-positivity.

Results Ninety-eight participants (50 men and 48 women) with a median age of 29 (range 20-52 years) were recruited. Overall, $67.4 \%$ (66 of 98) participants were positive. All participants reported a history of oral sex. Participants from a non-White ethnic group were more likely to be oral HPV-positive than Whites $(63.1 \%$ vs. $92.9 \%, \mathrm{p}=0.03)$ and those who engaged in open mouth/deep kissing in the last $24 \mathrm{~h}$ were also more likely to be oral HPV-positive than those who did not $(86.2 \%$ vs. $59.7 \%, p=0.01)$. No statistically significant associations were found with recent history of oral sex, smoking, alcohol and cannabis use, or lifetime number of sexual partners.

Conclusion Oral HPV infection is common among STI clinic attendees. It is unclear whether these are transient oral HPV infections or true persistent infections with oncogenic potential. Our limited data suggest that recent open mouth/deep kissing behaviour is associated with transmission of oral HPV.

\section{P236 IS ANNUAL CERVICAL CYTOLOGY IN HIV POSITIVE WOMEN JUSTIFIED IN THE ERA OF HPV TESTING? A 2-YEAR STUDY IN A DISTRICT GENERAL HOSPITAL}

Nisha Pal, Mamatha Odhuru*, Noreen Desmond. Berkshire Healthcare Foundation Trust, Garden Clinic, Slough, Berkshire, UK

\subsection{6/sextrans-2015-052126.278}

Background/introduction As per guidelines all HIV positive women have annual cytology irrespective of their CD4 count, viral load, and antiretroviral therapy. Smear tests are often cumbersome and most patients dislike annual smears. There is a lot of administration and cost involved in screening these women on an annual basis.

Aim(s)/objectives We looked at cervical cytology results of our HIV positive cohort for 2 years in the era of HPV testing and found some interesting results.

Methods Data collected on excel sheet and analysed.

Results Total of 153 cases was reviewed for over 2 years. 123/ 153 had negative HPV test. 30/153 had positive HPV test. 


\begin{tabular}{lll}
\hline $\begin{array}{l}\text { Negative cytology with HR HPV } \\
\text { detected }\end{array}$ & $5 / 30$ & Referred for colposcopy \\
$\begin{array}{l}\text { Mild /low grade dyskaryosis } \\
\text { Borderline changes }\end{array}$ & $\begin{array}{l}17 / 30 \\
6 / 30\end{array}$ & $\begin{array}{l}\text { Repeat smear in } 6 \text { months. } \\
\text { 3/6 HR HPV detected were referred for } \\
\text { colposcopy, } \\
3 / 6 \text { HPV not detected had repeat smear in } \\
6 \text { months. }\end{array}$ \\
Moderate dyskaryosis & $1 / 30$ & HR HPV detected, referred for colposcopy \\
Severe dyskaryosis & $1 / 30$ & HR HPV detected, referred for colposcopy \\
\hline
\end{tabular}

Out of the 30 with HR HPV: 5/30 was not on ARV. 25/30 on ARV had HIV VL <50 cpm. Age range from 28-62 years. 22/30 was Black African. 6/30 was white UK.

Conclusions Women with HIV infection who engage in medical care are usually on antiretroviral therapy and are virologically suppressed. The patients with HR HPV were followed up with colposcopy and continue to have annual smears. Patients with negative smear results who are HR HPV negative can be screened as per the normal population.c

\section{P237 HEPATITIS C AMONG MEN WHO HAVE SEX WITH MEN IN GREATER MANCHESTER - THE BASELINE SURVEY}

${ }^{1}$ Georgina Ireland*, ${ }^{2}$ Chris Ward, ${ }^{3}$ Sameena Ahmad, ${ }^{4}$ Ben Goorney, ${ }^{5}$ Steve Higgins, ${ }^{4}$ Catherine Stewart, ' Sam Lattimore, ${ }^{2}$ Vincent Lee. ${ }^{1}$ Public Health England, London, UK; ${ }^{2}$ Central Manchester University Hospitals, Greater Manchester, UK; ${ }^{3}$ University Hospital of South Manchester, Greater Manchester, UK; ${ }^{4}$ Salford Royal NHS Foundation Trust, Greater Manchester, UK; ${ }^{5}$ Pennine Acute Hospitals NHS Trust, Greater Manchester, UK

\subsection{6/sextrans-2015-052126.279}

Background/introduction The number of HIV affected men who have sex with men (MSM) co-infected with hepatitis C (HCV) continues to rise, driven by high risk sexual practice.

Aim(s)/objectives To determine HCV burden and associated risk behaviours among MSM in Greater Manchester.

Methods Between April and October 2014, all MSM attending four GUM clinics were asked to complete a risk assessment questionnaire and HCV screening was offered.

Results There were significant differences in risk behaviour between HIV positive and HIV negative MSM ( $p<0.05)$. Certain risk behaviours were strongly associated with HCV acquisition including: unprotected anal sex, sex with known HCV partners, fisting, group sex, 'slamming' and recreational drug use $(\mathrm{p}<0.002)$.

Discussion/conclusion Our study shows HIV positive MSM have significantly different sexual behaviour which may explain the higher HCV burden. However, HCV was found in HIV negative MSM engaging in high risk sexual practices. All MSM attending sexual health clinics must have a risk assessment and HCV screening should be offered based on the risk. Further studies are warranted to look at the difference in HCV transmission according to the HIV status.

\section{P238 HEPATITIS C TESTING IN MSM - ARE WE ASKING THE RIGHT QUESTIONS?}

Brenton Wait, Rachel Coyle*, lain Reeves, Tristan Barber. Homerton University Hospital, London, UK

\section{$10.1136 /$ sextrans-2015-052126.280}

Background Concern regarding high rates of hepatitis C infection in sub-groups of MSM may warrant targeted testing.
Aim We examine whether we routinely collect the necessary information from MSM to identify those at risk and target hepatitis $\mathrm{C}$ testing, and assess whether our concerns about emerging risk factors for hepatitis $\mathrm{C}$ are implicated in new diagnoses in our cohort.

Methods Notes audit of all MSM GUM attendances during November 2013 assessed documentation of fisting, rectal bleeding with sex, group sex, and drug use, as well as hepatitis testing. Notes of all patients coded for hepatitis $\mathrm{C}$ infection during 2011-2013 were examined to assess risk factors for hepatitis C infection.

Results 147 MSM attendances were reviewed. The proportion of men asked about specific risk factors was: drug use (18\%), rectal bleeding (1\%), group sex (1\%), fisting (1\%). 8\% MSM had hepatitis $\mathrm{C}$ screens, none with traditional risk factors. Over 3 years, 46 patients were coded for hepatitis C. $34 \%$ of these were new infections. $33 \%$ were HIV positive, $48 \%$ had injected drugs (41\% no documentation), $22 \%$ had hepatitis C positive partners, $11 \%$ were sex workers.

Discussion/conclusion Drug use and high risk sexual practices were not always fully recorded in our sample. Testing rates were low and did not seem to relate to identifiable risks. We identified few cases of new infection, largely limited to patients with traditional risk factors. It is not clear if better recording of risk factors would lead to increased Hepatitis $\mathrm{C}$ testing or diagnosis.

\section{Category: Women and children}

\section{P239 DOES SERVICE INTEGRATION IMPROVE THE SEXUAL AND REPRODUCTIVE HEALTHCARE OF HIV POSITIVE WOMEN?}

Sally Wielding*. Chalmers Centre, NHS Lothian, Edinburgh, UK

\section{$10.1136 /$ sextrans-2015-052126.281}

Background NHS Lothian Genitourinary Medicine (GUM) and Sexual and Reproductive Healthcare (SRH) services integrated in June 2011. Contraceptive use, pregnancies and uptake of annual cervical cytology were audited in a cohort of HIV positive women pre- and post-integration of services.

Aims To assess whether the SRH of HIV positive women has improved after integration of services, and to guide further service improvements.

Methods Case notes and electronic data recording system entries were interrogated for the 5 years preceding integration of services and the 3 years following integration.

Results Contraception: Pre-integration $24.9 \%$ of 70 women with contraceptive needs were on effective prescriptions. Postintegration this proportion rose to $39.3 \%$ of 74 women.

Pregnancies: In the 5 years pre-integration 32 women had 42 pregnancies. $47.6 \%$ of these pregnancies were unplanned (UP). In the 3 years post-integration 13 women had a total of 18 pregnancies, $50 \%$ were UP pregnancies.

Cervical cytology: Pre-integration $47.3 \%$ of those eligible had a cervical cytology result documented within the last year, which improved to $74.6 \%$.

Conclusion Contraceptive provision improved after service integration although there remained fewer than $40 \%$ of women using a suitable method. Despite this improvement, UP pregnancy rates did not fall significantly. In a cohort of women attending an integrated service regularly, who are known to have an infection which can be vertically transmitted, it is 